\title{
CHARACTERIZATION OF THE RANGE OF THE HILBERT TRANSFORM FOR BOUNDED BANDLIMITED SIGNALS AND APPLICATIONS
}

\author{
Holger Boche* \\ Technische Universität München \\ Lehrstuhl für Theoretische Informationstechnik
}

\author{
Ullrich J. Mönich ${ }^{\dagger}$ \\ Massachusetts Institute of Technology \\ Research Laboratory of Electronics
}

\begin{abstract}
Recently, a new constructive formula for the calculation of the Hilbert transform of bounded bandlimited signals was found. In this paper we use that formula to analyze the properties of the Hilbert transform. We further present a Fefferman-Stein-type decomposition theorem for bandlimited signals in $\mathrm{BMO}(\mathbb{R})$, i.e., bandlimited signals of bounded mean oscillation. Based on this decomposition we characterize the range of the Hilbert transform and derive properties of general bandlimited signals in $\mathrm{BMO}(\mathbb{R})$. We show the boundedness of bandpass signals in $\mathrm{BMO}(\mathbb{R})$ and the boundedness of the derivative of bandlimited signals in $\mathrm{BMO}(\mathbb{R})$. We further find the maximum growth of the Hilbert transform of bounded bandlimited signals.
\end{abstract}

Index Terms - Hilbert transform, bounded mean oscillation, growth behavior, bandlimited signal, bandpass signal

\section{INTRODUCTION}

The classical principal value integral definition of the Hilbert transform

$$
\begin{aligned}
(H f)(t) & =\frac{1}{\pi} \text { V.P. } \int_{-\infty}^{\infty} \frac{f(\tau)}{t-\tau} \mathrm{d} \tau=\frac{1}{\pi} \lim _{\epsilon \rightarrow 0} \int_{\epsilon \leq|t-\tau| \leq \frac{1}{\epsilon}} \frac{f(\tau)}{t-\tau} \mathrm{d} \tau \\
& =\frac{1}{\pi} \lim _{\epsilon \rightarrow 0}\left(\int_{t-\frac{1}{\epsilon}}^{t-\epsilon} \frac{f(\tau)}{t-\tau} \mathrm{d} \tau+\int_{t+\epsilon}^{t+\frac{1}{\epsilon}} \frac{f(\tau)}{t-\tau} \mathrm{d} \tau\right) .
\end{aligned}
$$

cannot be used to define the Hilbert transform for bounded bandlimited signals, because there are bounded bandlimited signals for which the principal value integral (1) diverges for all $t \in \mathbb{R}[1]$.

Bounded bandlimited signals are important in practical applications, for example in wireless communication systems, where the peak-to-average power ratio (PAPR) of signals is an essential quantity [2], or in the decomposition of signals in elementary components, as described in [3].

However, the convergence problem of (1) for bounded bandlimited signals does not mean that the Hilbert transform cannot be meaningfully defined for this space. Based on the abstract $\mathcal{H}^{1}$ $\mathrm{BMO}(\mathbb{R})$ duality theory (for a definition of $\mathcal{H}^{1}$ and $\mathrm{BMO}(\mathbb{R})$ see next section) it is possible to define the Hilbert transform for arbitrary bounded signals. A major drawback of this definition is its

\footnotetext{
* This work was partly supported by the German Research Foundation (DFG) under grant BO 1734/13-2.

${ }^{\dagger}$ U. Mönich was supported by the German Research Foundation (DFG) under grant MO 2572/1-1. U. Mönich was with the Lehrstuhl für Theoretische Informationstechnik, Technische Universität München, when the majority of this work was completed.
}

abstract nature; the duality theory itself provides no formula for the calculation of the Hilbert transform. The lack of such a formula makes a further analysis of the properties of the Hilbert transform difficult. Recently, some advance has been made to eliminate this problem. In [1, 4] an explicit formula for the calculation of the Hilbert was found for the space of bounded bandlimited signals.

Based on this formula we will analyze the properties of the Hilbert transform of bounded bandlimited signals. We achieve a complete understanding of the image of the space of bounded bandlimited signals under the Hilbert transform and characterize the structure of bandlimited $\mathrm{BMO}(\mathbb{R})$-signals. Based on the findings, we further provide an upper bound for the $L^{\infty}(\mathbb{R})$-norm of the derivative of bandlimited $\mathrm{BMO}(\mathbb{R})$-signals.

\section{NOTATION}

Let $\hat{f}$ denote the Fourier transform of a function $f . L^{p}(\mathbb{R}), 1 \leq p<$ $\infty$, is the space of all $p$ th-power Lebesgue integrable functions on $\mathbb{R}$, with the usual norm $\|\cdot\|_{p}$, and $L^{\infty}(\mathbb{R})$ is the space of all functions for which the essential supremum norm $\|\cdot\|_{\infty}$ is finite. $\mathcal{H}^{1}$ denotes Hardy space of all functions $f \in L^{1}(\mathbb{R})$ for which $H f \in L^{1}(\mathbb{R})$. For $0<\sigma<\infty$ let $\mathcal{B}_{\sigma}$ be the set of all entire functions $f$ with the property that for all $\epsilon>0$ there exists a constant $C(\epsilon)$ with $|f(z)| \leq C(\epsilon) \exp ((\sigma+\epsilon)|z|)$ for all $z \in \mathbb{C}$. The Bernstein space $\mathcal{B}_{\sigma}^{p}, 1 \leq p \leq \infty$, consists of all functions in $\mathcal{B}_{\sigma}$, whose restriction to the real line is in $L^{p}(\mathbb{R})$. The norm for $\mathcal{B}_{\sigma}^{p}$ is given by the $L^{p}$-norm on the real line, i.e., $\|\cdot\|_{\mathcal{B}_{\sigma}^{p}}=\|\cdot\|_{p}$. A function in $\mathcal{B}_{\sigma}^{p}$ is called bandlimited to $\sigma$, and $\mathcal{B}_{\sigma}^{\infty}$ is the space of bandlimited functions that are bounded on the real axis. We call a function in $\mathcal{B}_{\pi}^{\infty}$ bounded bandlimited signal.

A function $f: \mathbb{R} \rightarrow \mathbb{C}$ is said to belong to $\operatorname{BMO}(\mathbb{R})$, provided that it is locally in $L^{1}(\mathbb{R})$ and $\frac{1}{|I|} \int_{I}\left|f(t)-m_{I}(f)\right| \mathrm{d} t \leq C_{1}$ for all bounded intervals $I$, where $m_{I}(f):=\frac{1}{|I|} \int_{I} f(t) \mathrm{d} t$ and the constant $C_{1}$ is independent of $I .|I|$ denotes the Lebesgue measure if the set $I$. We further define

$$
\|f\|_{\mathrm{BMO}(\mathbb{R})}=\sup _{I} \frac{1}{|I|} \int_{I}\left|f(t)-m_{I}(f)\right| \mathrm{d} t,
$$

where the supremum is over all bounded intervals $I$. Note that $\|\cdot\|_{\mathrm{BMO}(\mathbb{R})}$ is actually a seminorm, because we have $\|c\|_{\mathrm{BMO}(\mathbb{R})}=0$ for all constants $c \in \mathbb{C}$. Further, we denote by $\mathrm{BMO}_{\pi}$ the space of all functions in $\mathcal{B}_{\pi}$ that are in $\operatorname{BMO}(\mathbb{R})$ when restricted to the real axis.

\section{THE HILBERT TRANSFORM FOR $\mathcal{B}_{\pi}^{\infty}$}

Despite the convergence problems of the principal value integral, there is a way to define the Hilbert transform for signals in $\mathcal{B}_{\pi}^{\infty}$. 
This definition uses Fefferman's duality theorem [5], which states that the dual space of $\mathcal{H}^{1}$ is $\mathrm{BMO}(\mathbb{R})$. In this definition the Hilbert transform $\mathfrak{H} f$ of a bounded bandlimited signal $f \in \mathcal{B}_{\pi}^{\infty}$ is a signal in the space $\operatorname{BMO}(\mathbb{R})$. However, due to technical reasons, the Hilbert transform is unique only up to an arbitrary additive constant $C_{\mathrm{BMO}}$. In a strict mathematical sense, the Hilbert transform in this definition is not a single signal but an equivalence class that contains all signals that differ only by an additive constant. By $[f]$ we denote the equivalence class $[f]=\{g \in \mathrm{BMO}(\mathbb{R})$ : $g$ and $f$ differ only by an additive constant. $\}$. This is the reason why use a different notation for the Hilbert transform. We use $\mathfrak{H} f$ instead of $H$, which was used in the introduction for the classical Hilbert transform. For technical details see $[4,1]$.

In addition to this rather abstract definition, there also exists a constructive approach for the calculation of the Hilbert transform for signals in $\mathcal{B}_{\pi}^{\infty}$. This approach was presented in [4]. We briefly review the most important facts.

Consider the linear time-invariant (LTI) system defined by

$$
Q^{\mathrm{E}} f=\sum_{k=-\infty}^{\infty} a_{-k} f(\cdot-k)
$$

where the coefficients $a_{k}, k \in \mathbb{Z}$, are given by

$$
a_{k}=\frac{1}{2 \pi} \int_{-\pi}^{\pi}|\omega| \mathrm{e}^{i \omega k} \mathrm{~d} \omega= \begin{cases}\frac{\pi}{2}, & k=0, \\ \frac{(-1)^{k}-1}{\pi k^{2}}, & k \neq 0 .\end{cases}
$$

It can be shown (see [4] for details) that the mapping (2) defines a bounded linear operator $Q^{\mathrm{E}}: \mathcal{B}_{\pi}^{\infty} \rightarrow \mathcal{B}_{\pi}^{\infty}$ with norm $\left\|Q^{\mathrm{E}}\right\|=\pi$. Hence, for every $f \in \mathcal{B}_{\pi}^{\infty}$, the operator $\mathfrak{I}$ given by

$$
(\mathfrak{I} f)(t)=\int_{0}^{t}\left(Q^{\mathrm{E}} f\right)(\tau) \mathrm{d} \tau, \quad t \in \mathbb{R},
$$

is well defined. In [1] it was shown that $\mathfrak{I} f$ is a representative of the equivalence class $\mathfrak{H} f$. Loosely speaking, $\mathfrak{I} f$ is the desired Hilbert transform $\mathfrak{H} f$.

Theorem 1. Let $f \in \mathcal{B}_{\pi}^{\infty}$. Then we have $\mathfrak{H} f=[\mathfrak{I} f]$. Further the Hilbert transform is again bandlimited because $\mathfrak{I} f \in B M O_{\pi}$.

Theorem 1 is very useful, because it enables us to compute the Hilbert transform of bounded bandlimited signals in $\mathcal{B}_{\pi}^{\infty}$ by using the constructive formula (4), instead of using the abstract definition which is based on the the $\mathcal{H}^{1}-\mathrm{BMO}(\mathbb{R})$ duality. Next, we will use this formula to derive properties of the Hilbert transform and of general $\mathrm{BMO}_{\pi}$-signals.

\section{A FEFFERMAN-STEIN-TYPE THEOREM FOR BMO ${ }_{\pi}$}

Fefferman's decomposition theorem, which states that an arbitrary $\operatorname{BMO}(\mathbb{R})$-signal can be decomposed into the sum of a $L^{\infty}(\mathbb{R})$ signal and the Hilbert transform of a $L^{\infty}(\mathbb{R})$-signal. For signals in $f \in \mathrm{BMO}_{\pi}$, i.e., signals in $\mathrm{BMO}(\mathbb{R})$ that are additionally bandlimited, the above decomposition is of course also possible because $\mathrm{BMO}_{\pi} \subset \mathrm{BMO}(\mathbb{R})$. From Fefferman's decomposition theorem we know that the two signals that arise in the decomposition are in $L^{\infty}(\mathbb{R})$. However, since the signal $f$ is additionally bandlimited, it is reasonable to ask whether the decomposition can be performed in such a way that the two signals in the decomposition are additionally bandlimited, i.e., in $\mathcal{B}_{\pi}^{\infty}$. The next theorem answers this question in the affirmative.
Theorem 2. There exists a constant $C_{2}>0$ such that for all $f \in$ $B M O_{\pi}$ there exist two signals $f_{1}, f_{2} \in \mathcal{B}_{\pi}^{\infty}$ and a constant $\alpha$ such that $f=f_{1}+\mathfrak{H} f_{2}+\alpha$ and $\left\|f_{1}\right\|_{\infty} \leq C_{2}\|f\|_{B M O(\mathbb{R})},\left\|f_{2}\right\|_{\infty} \leq$ $C_{2}\|f\|_{B M O(\mathbb{R})}$.

The proof of Theorem 2 is omitted due to space constraints.

The following corollary of Theorem 2 is a structure result for $\mathrm{BMO}_{\pi}$-signals.

Corollary 1. For all $0<\hat{\beta} \leq 1$ there exists a constant $C_{3}$ such that for all $f \in B M O_{\pi}$ there exist two functions $f_{3} \in \mathcal{B}_{\pi}^{\infty}$ and $f_{4} \in B M O_{\hat{\beta} \pi}$ and a constant $\alpha$ such that $f=f_{3}+f_{4}+\alpha$ and $\left\|f_{3}\right\|_{\infty} \leq C_{3}(\hat{\beta})\|f\|_{B M O(\mathbb{R})},\left\|f_{4}\right\|_{B M O(\mathbb{R})} \leq C_{3}(\hat{\beta})\|f\|_{B M O(\mathbb{R})}$.

Corollary 1 shows that every $\mathrm{BMO}_{\pi}$-signal $f$ can be decomposed into the sum of a $\mathcal{B}_{\pi}^{\infty}$-signal $f_{3}$ and a low-pass $\mathrm{BMO}_{\hat{\beta} \pi}$-signal $f_{4}$. The bandwidth of the signal $f_{4}$ can be arbitrarily low, i.e., every number $0<\hat{\beta} \leq \pi$ can be chosen. However, a lower bandwidth of $f_{4}$ will lead in general to a larger constant $C_{3}(\hat{\beta})$ for the peak value of $f_{3}$. Corollary 1 will play an important role in Section 8 , where we analyze bandpass signals in $\mathrm{BMO}(\mathbb{R})$.

From a mathematical point of view Theorem 2 is interesting, because it is the direct analogon of Fefferman's decomposition theorem for bandlimited $\mathrm{BMO}(\mathbb{R})$-signals.

\section{RANGE OF THE HILBERT TRANSFORM}

With the results in the previous section, we can characterize the range of the Hilbert transform, i.e. the image of $\mathcal{B}_{\pi}^{\infty}$ under $\mathfrak{H}$. From the definition of $\mathfrak{H}$ and Theorem 1 we know that the range is a subset of $\mathrm{BMO}_{\pi}$. Now, Theorem 2 shows that the range is essentially $\mathrm{BMO}_{\pi}$ in the sense that every signal in $\mathrm{BMO}_{\pi}$ is the Hilbert transform of some signal in $\mathcal{B}_{\pi}^{\infty}$ modulo a signal in $\mathcal{B}_{\pi}^{\infty}$. More precisely, for all $g \in \mathrm{BMO}_{\pi}$ there exists a signal $f \in \mathcal{B}_{\pi}^{\infty}$ such that $\|\mathfrak{H} f-g\|_{\infty}<\infty$. Of course $g$ does not need to be bounded. However, since $\|\mathfrak{H} f-g\|_{\infty}<\infty$, we see that the divergence behavior of $g$ is "created" exactly by $\mathfrak{H} f$.

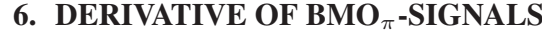

For signals $f \in \mathcal{B}_{\pi}^{\infty}$ there is the well-known Bernstein inequality,

$$
\left\|f^{\prime}\right\|_{\infty} \leq \pi\|f\|_{\infty}
$$

stating that the derivative of a bounded bandlimited signal is again bounded and that the peak value of the derivative is smaller than or equal to a constant factor times the peak value of the signal itself [6, p. 49]. For general signals in $\mathrm{BMO}_{\pi}$ the above inequality (5) is meaningless, because for unbounded signals in $\mathrm{BMO}_{\pi}$ the right hand side of (5) is infinity. A priori it is not clear whether the derivative of a signal in $\mathrm{BMO}_{\pi}$ is bounded.

The next theorem shows that every signal in $\mathrm{BMO}_{\pi}$ has a bounded derivative. Moreover, the peak value of the derivative is smaller than or equal to a constant factor times the $\mathrm{BMO}(\mathbb{R})$-norm of the signal. Thus, there exists an inequality similar (5) for signals in $\mathrm{BMO}_{\pi}$

Theorem 3. There exists a constant $C_{4}$ such that for all $f \in B M O_{\pi}$ we have $\left\|f^{\prime}\right\|_{\infty} \leq C_{4}\|f\|_{B M O(\mathbb{R})}$.

Theorem 3 is remarkable because it shows that signals in $\mathrm{BMO}_{\pi}$, i.e., bandlimited $\mathrm{BMO}(\mathbb{R})$-signals, which can be unbounded themselves, always have a bounded derivative. This implies that the amount of oscillation of $\mathrm{BMO}_{\pi}$-signals is limited. 
Proof. According to Theorem 2 there exists a constant $C_{2}>0$ such that for all $f \in \mathrm{BMO}_{\pi}$ there exist two signals $f_{1}, f_{2} \in \mathcal{B}_{\pi}^{\infty}$ and a constant $\alpha$ such that $f=f_{1}+\mathfrak{H} f_{2}+\alpha$ and $\left\|f_{1}\right\|_{\infty} \leq C_{2}\|f\|_{\mathrm{BMO}(\mathbb{R})}$, $\left\|f_{2}\right\|_{\infty} \leq C_{2}\|f\|_{\mathrm{BMO}(\mathbb{R})}$. It follows that $f^{\prime}=f_{1}^{\prime}+Q^{\mathrm{E}} f_{2}$ and consequently that

$$
\begin{aligned}
\left\|f^{\prime}\right\|_{\infty} & \leq\left\|f_{1}^{\prime}\right\|_{\infty}+\left\|Q^{\mathrm{E}} f_{2}\right\|_{\infty} \\
& \leq \pi\left\|f_{1}\right\|_{\infty}+\pi\left\|f_{2}\right\|_{\infty} \\
& \leq 2 \pi C_{2}\|f\|_{\mathrm{BMO}(\mathbb{R})}
\end{aligned}
$$

for all $f \in \mathrm{BMO}_{\pi}$. In the second inequality of (6) we used Bernstein's inequality [6, p. 49].

\section{PEAK VALUE BEHAVIOR ON FINITE INTERVALS}

The peak value of signals is important for many applications, e.g., for the hardware design in mobile communications. For a recent overview, see [2]. The growth behavior of the Hilbert transform of signals in $\mathcal{B}_{\pi}^{\infty}$ was studied in [7]. For all $f \in \mathcal{B}_{\pi}^{\infty}$, we have the upper bound

$$
\begin{aligned}
|(\Im f)(t)| & \leq \int_{0}^{t}\left|\left(Q^{\mathrm{E}} f\right)(\tau)\right| \mathrm{d} \tau \\
& \leq\left\|Q^{\mathrm{E}} f\right\|_{\infty}|t| \\
& \leq \pi\|f\|_{\infty}|t|, \quad t \in \mathbb{R},
\end{aligned}
$$

which shows that the asymptotic growth of the Hilbert transform $\mathfrak{H} f$ of signals $f \in \mathcal{B}_{\pi}^{\infty}$ is at most linear. More precisely, for all $f \in \mathcal{B}_{\pi}^{\infty}$ there exists a signal $g \in \mathrm{BMO}(\mathbb{R})$ such that $\mathfrak{H} f=[g]$ and $g(t)=O(t)$.

On the other hand, using the identity (4), it can be shown that for the $\mathcal{B}_{\pi}^{\infty}$-signal

$$
f_{1}(t)=\frac{2}{\pi} \int_{0}^{\pi} \frac{\sin (\omega t)}{\omega} \mathrm{d} \omega
$$

we have

$$
\left|\left(\Im f_{1}\right)(t)\right| \geq \frac{2}{\pi}\left(\log (|t|)-\frac{\pi^{2}}{4}-1-\frac{1}{\pi}\right)
$$

for all $t \in \mathbb{R}$ with $|t| \geq 1$. Thus, there are signals $f \in \mathcal{B}_{\pi}^{\infty}$, such that the growth of the Hilbert transform $\mathfrak{H} f$ is logarithmic, in the sense that there exists a signal $g \in \operatorname{BMO}(\mathbb{R})$ such that $\mathfrak{H} f=[g]$ and $g(t)=\Omega(\log (t))$.

From this the question arises whether the asymptotically logarithmic growth is actually the maximum possible growth, i.e., whether the upper bound (7) can be improved. The next theorem, which was published in [7], gives a positive answer.

Theorem 4. There exist two positive constants $C_{5}$ and $C_{6}$ such that for all $f \in \mathcal{B}_{\pi}^{\infty}$ and all $t \in \mathbb{R}$ we have

$$
|(\Im f)(t)| \leq C_{5} \log (1+|t|)\|f\|_{\infty}+C_{6}\|f\|_{\infty} .
$$

Thanks to Theorem 4 and the structure result for $\mathrm{BMO}_{\pi}$, which was given in Theorem 2, we are able to derive a growth estimate for arbitrary signals in $\mathrm{BMO}_{\sigma}, 0<\sigma<\infty$.

Theorem 5. Let $f \in B M O_{\sigma}, 0<\sigma<\infty$. Then, for all $\gamma>\sigma$, there exists a constant $C_{7}$ such that

$$
|f(z)| \leq C_{7} \mathrm{e}^{\gamma|\operatorname{Im}(z)|} \log (2+|\operatorname{Re}(z)|)
$$

for all $z \in \mathbb{C}$.
Before we prove Theorem 5, we state a simple corollary and discuss its relation to Theorem 4 .

Corollary 2. Let $f \in B M O_{\pi}$. Then there exists a constant $C_{7}$ such that

$$
|f(t)| \leq C_{7} \log (2+|t|)
$$

for all $t \in \mathbb{R}$.

Theorem 4 has shown that the growth of the Hilbert transform of a signal in $\mathcal{B}_{\pi}^{\infty}$ is at most logarithmic, and Corollary 2 shows that the growth of an arbitrary signal in $\mathrm{BMO}_{\pi}$ is at most logarithmic. Since $\left\{\Im f: f \in \mathcal{B}_{\pi}^{\infty}\right\} \subset \mathrm{BMO}_{\pi}$, Corollary 2 is a generalization of Theorem 4 to the whole space $\mathrm{BMO}_{\pi}$.

Definition 1. Let $\mathcal{K}_{\sigma}, 0<\sigma<\infty$, denote the space of all functions $K \in \mathcal{B}_{\sigma}^{1}$, whose Fourier transform $\hat{K}$ is two times continuously differentiable. For $0 \leq \omega_{1}<\omega_{2}<\sigma<\infty$ let

$$
\mathcal{K}_{\sigma}\left(\omega_{1}, \omega_{2}\right)=\left\{K \in \mathcal{K}_{\sigma}: \hat{f}(\omega)=1 \text { for }|\omega| \in\left[\omega_{1}, \omega_{2}\right]\right\} .
$$

Sketch of the proof of Theorem 5. Let $f \in \mathrm{BMO}_{\sigma}, 0<\sigma<\infty$, and $\gamma$ satisfying $\sigma<\gamma<\infty$ be arbitrary but fixed. Since $f \in \mathcal{B}_{\sigma}$, there exists a constant $C_{8}$ such that

$$
\begin{aligned}
|f(z)| & \leq C_{8} \mathrm{e}^{\sigma|z|} \leq C_{8} \mathrm{e}^{\sigma(|\operatorname{Re}(z)|+|\operatorname{Im}(z)|} \\
& \leq C_{8} \mathrm{e}^{2 \sigma} \mathrm{e}^{\gamma|\operatorname{Im}(z)|}
\end{aligned}
$$

for all $z \in \mathbb{C}$ with $|\operatorname{Re}(z)| \leq 2$. Next, we deal with the case $|\operatorname{Re}(z)| \geq 2$. Choose some $K \in \mathcal{K}_{\gamma}(0, \sigma)$. Using integration by parts it can be shown that there exists a constant $C_{9}$ such that

$$
|K(z)| \leq C_{9} \frac{\mathrm{e}^{i \gamma|\operatorname{Im}(z)|}}{1+|z|^{2}}
$$

for all $z \in \mathbb{C}$. We further have $f(z)=\int_{-\infty}^{\infty} K(z-\tau) f(\tau) \mathrm{d} \tau$ for all $z \in \mathbb{C}$. Thus, it follows that

$$
|f(z)| \leq C_{9} \mathrm{e}^{i \gamma|\operatorname{Im}(z)|} \int_{-\infty}^{\infty} \frac{|f(\tau)|}{1+|z-\tau|^{2}} \mathrm{~d} \tau .
$$

Next, we analyze the integral on the right hand side of (11) for $|\operatorname{Re}(z)| \geq 2$. We use the abbreviation $t=\operatorname{Re}(z)$, and restrict ourselves to $t \geq 2$. The case $t \leq-2$ is treated analogously. Let $t \geq 2$ be arbitrary but fixed. Using Theorems 2 and 4 it can be shown after some lengthy but elementary calculation that there exists a constant $C_{10}$ such that

$$
\int_{-\infty}^{\infty} \frac{|f(\tau)|}{1+|t-\tau|^{2}} \mathrm{~d} \tau \leq C_{10} \log (2+t)
$$

Since the case $t \leq-2$ is treated analogously, it follows that

$$
\int_{-\infty}^{\infty} \frac{|f(\tau)|}{1+|z-\tau|^{2}} \mathrm{~d} \tau \leq C_{10} \log (2+|\operatorname{Re}(z)|)
$$

for all $z \in \mathbb{C}$ with $|\operatorname{Re}(z)| \geq 2$. This together with (11) completes the proof. 


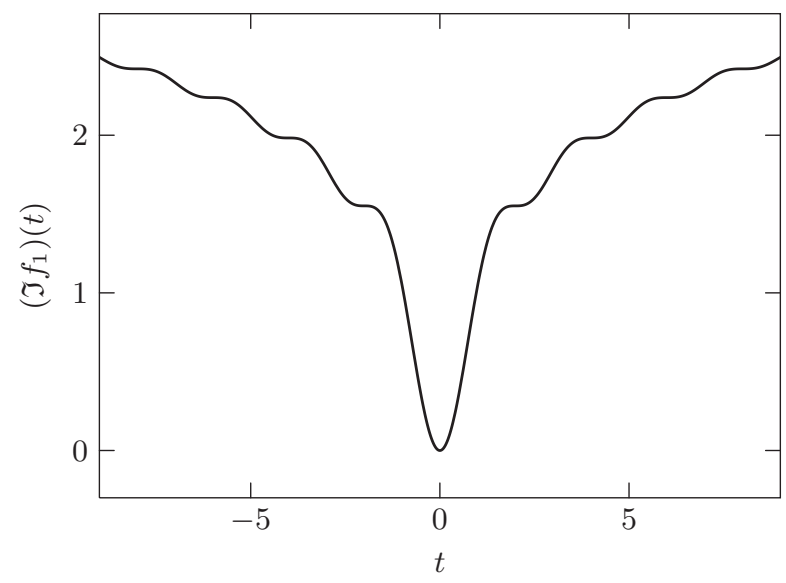

Fig. 1. Plot of the signal $\Im f_{1}$.

\section{BANDPASS SIGNALS IN BMO $(\mathbb{R})$}

In general neither $\mathrm{BMO}(\mathbb{R})$-signals nor bandpass signals are necessarily bounded. Let $f_{1}$ be the signal that was defined in (8). An example for an unbounded $\operatorname{BMO}(\mathbb{R})$-signal is $\mathfrak{I} f_{1}$, which is plotted in Fig. 1, and an example for an unbounded bandpass signal is $t \sin (\pi t)$. In this section we treat bandpass signals in $\mathrm{BMO}(\mathbb{R})$, i.e., $\mathrm{BMO}(\mathbb{R})$-signals that are additionally bandpass signals, and show that those signals are always bounded on the real axis.

Definition 2. Let $\mathrm{BMO}_{\left[\omega_{1}, \omega_{2}\right]}$ be the space of all signals $f \in$ $\operatorname{BMO}(\mathbb{R})$ that fulfill $f(t)=\int_{-\infty}^{\infty} f(\tau) K(t-\tau) \mathrm{d} \tau$ for all $t \in \mathbb{R}$ and all $K \in \mathcal{K}_{\sigma}\left(\omega_{1}, \omega_{2}\right), \sigma>\omega_{2}$.

Theorem 6. Let $0<\omega_{1}<\omega_{2}<\infty$ and $f \in B M O_{\left[\omega_{1}, \omega_{2}\right]}$. Then we have $f \in \mathcal{B}_{\omega_{2}}^{\infty}$.

Proof. Let $0<\omega_{1}<\omega_{2}<\infty, f \in \mathrm{BMO}_{\left[\omega_{1}, \omega_{2}\right]}$, and $\sigma>\omega_{2}$ be arbitrary but fixed. Further, choose some $\gamma$ satisfying $0<\gamma<$ $\omega_{1}$. According to Corollary 1 there exist two signals $f_{3} \in \mathcal{B}_{\omega_{2}}^{\infty}$ and $f_{4} \in \mathrm{BMO}_{\gamma}$ and a constant $\alpha$ such that $f=f_{3}+f_{4}+\alpha$. Let $K_{1} \in \mathcal{K}_{\sigma}\left(0, \omega_{2}\right), K_{2} \in \mathcal{K}_{\omega_{1}}(0, \gamma)$, and $K=K_{1}-K_{2}$. Then we have

$$
\int_{-\infty}^{\infty} f_{4}(\tau) K_{1}(t-\tau) \mathrm{d} \tau=\int_{-\infty}^{\infty} f_{4}(\tau) K_{2}(t-\tau) \mathrm{d} \tau
$$

and it follows that

$$
\begin{aligned}
\int_{-\infty}^{\infty} & f_{4}(\tau) K(t-\tau) \mathrm{d} \tau \\
& =\int_{-\infty}^{\infty} f_{4}(\tau) K_{1}(t-\tau) \mathrm{d} \tau-\int_{-\infty}^{\infty} f_{4}(\tau) K_{2}(t-\tau) \mathrm{d} \tau \\
& =0
\end{aligned}
$$

Thus, we have

$$
\begin{aligned}
f(t)= & \int_{-\infty}^{\infty} f(\tau) K(t-\tau) \mathrm{d} \tau \\
= & \int_{-\infty}^{\infty} f_{3}(\tau) K(t-\tau) \mathrm{d} \tau+\int_{-\infty}^{\infty} f_{4}(\tau) K(t-\tau) \mathrm{d} \tau \\
& +\int_{-\infty}^{\infty} \alpha K(t-\tau) \mathrm{d} \tau \\
= & \int_{-\infty}^{\infty} f_{3}(\tau) K(t-\tau) \mathrm{d} \tau
\end{aligned}
$$

where the first equality follows from our assumption that $f \in$ $\mathrm{BMO}_{\left[\omega_{1}, \omega_{2}\right]}$ and the fact that $K \in \mathcal{K}_{\sigma}\left(\omega_{1}, \omega_{2}\right)$. Since

$$
\int_{-\infty}^{\infty} f_{3}(\tau) K(\cdot-\tau) \mathrm{d} \tau \in \mathcal{B}_{\sigma}^{\infty}
$$

and $\sigma>\omega_{2}$ was arbitrary, it follows that $f \in \mathcal{B}_{\omega_{2}}^{\infty}$.

\section{RELATION TO PRIOR WORK}

Long time it was believed "that an arbitrary bounded bandlimited function does not have a Hilbert transform..." [8]. However, based on the abstract $\mathcal{H}^{1}-\mathrm{BMO}(\mathbb{R})$ duality theory it is possible to define the Hilbert transform for those signals. The main drawback of this definition is abstract nature, which provides no formula for the calculation. In [1, 4] a constructive approach was presented that makes it possible to calculate the Hilbert transform with a simple formula. Using this formula, we were able to characterize the range of the Hilbert transform and derive several interesting properties of the Hilbert transform and general $\mathrm{BMO}_{\pi}$-signals. The Hilbert transform is an important operation in communication theory and signal processing. For example, the "analytical signal", which was used by Dennis Gabor in his "Theory of Communication" [9], is based on the Hilbert transform. Further concepts in which the Hilbert transform is an integral part, are the instantaneous amplitude, phase, and frequency of a signal and the theory of modulation [8].

\section{REFERENCES}

[1] Holger Boche and Ullrich J. Mönich, "On the Hilbert transform of bounded bandlimited signals," Problems of Information Transmission, vol. 48, no. 3, pp. 217-238, 2012.

[2] Gerhard Wunder, Robert F.H. Fischer, Holger Boche, Simon Litsyn, and Jong-Seon No, "The PAPR problem in OFDM transmission: New directions for a long-lasting problem," IEEE Signal Processing Magazine, 2012, to be published.

[3] Norden E. Huang, Zheng Shen, Steven R. Long, Manli C. Wu, Hsing H. Shih, Quanan Zheng, Nai-Chyuan Yen, Chi Chao Tung, and Henry H. Liu, "The empirical mode decomposition and the Hilbert spectrum for nonlinear and non-stationary time series analysis," Proceedings of the Royal Society of London. Series A: Mathematical, Physical and Engineering Sciences, vol. 454, no. 1971, pp. 903-995, Mar. 1998.

[4] Holger Boche and Ullrich J. Mönich, "Extension of the Hilbert transform," in Proceedings of the IEEE International Conference on Acoustics, Speech, and Signal Processing (ICASSP '12), Mar. 2012, pp. 3697-3700.

[5] Charles Fefferman, "Characterization of bounded mean oscillation," Bull. of the AMS, vol. 77, no. 4, pp. 587-588, July 1971.

[6] John R. Higgins, Sampling Theory in Fourier and Signal Analysis - Foundations, Oxford University Press, 1996.

[7] Holger Boche and Ullrich J. Mönich, "Solution of the peak value problem for Gabor's theory of communication," in Proceedings of the 2012 International Symposium on Signals, Systems and Electronics, 2012.

[8] B. F. Logan, Jr., "Theory of analytic modulation systems," Bell System Technical Journal, vol. 57, no. 3, pp. 491-576, Mar. 1978.

[9] Dennis Gabor, "Theory of communication," Journal of the Institute of Electrical Engineers, vol. 93, no. 3, pp. 429-457, Nov. 1946. 